\title{
The Role of AhR-regulated Nrf2 induced by Benzo[a]pyrene in Retinal Pigment Epithelium Cells
}

\author{
Yen-Ling Kuo ${ }^{1}$, Chi-Hao Tsai ${ }^{1}$, Yu-Wen Cheng ${ }^{2}$, Jaw-Jou Kang ${ }^{1}$ \\ ${ }^{I}$ National Taiwan University College of Medicine Graduate Institute of Toxicology, Taiwan, ${ }^{2}$ Taipei Medical University \\ College of Medicine, Department of Pharmacpology, Taiwan
}

\section{Background:}

Age-related macular degeneration (AMD) is a disease associated with environmental factors. In recent years, studies have shown that smoking increases the chances of developing AMD, and it may be related to the reactive oxygen species (ROS) attack on the retinal pigment epithelium (RPE) cells. However, nuclear factor E2-related factor 2 (Nrf2), a transcription factor, will activate anti-oxidative gene translation and protect cells from ROS damage. Further previous studies showed that Nrf2 may be regulated by aryl hydrocarbon receptor (AhR). This regulatory mechanism might be used for the therapy for AMD. Therefore, the aim of this research is to study the interaction between AhR and Nrf2 in RPE cells by AhR ligand treatment.

Methods:

We used Benzo [a] pyrene (B[a]P), one of the compounds widely presenting in cigarettes, as a ligand to induce AhR activity and it would produce ROS after AhR metabolic processes. ARPE-19 cells line were treated with B[a]P and cotreated with AhR inhibitor (CH223191) for observing survival rate, mRNA and protein expression of RPE cells by MTT assay, RT-PCR and Western blot, respectively. ROS level was quantitative by DCFH-DA assay. Furthermore, CHIP assay was used to prove if AhR interacts with Nrf2 promoter region directly.

Results:

The results showed that $\mathrm{B}[\mathrm{a}] \mathrm{P}$ didn't affect the survival rate of RPE cells, presumably protected by the Nrf2-related antioxidant mechanism. And AhR regulated Nrf2 by two pathways, one is that it may metabolic ligand which then generate ROS inhibiting Nrf2 degradation. The other is that ligand promoting AhR nuclear import and binding to the promoter region of Nrf2 results in an increase of Nrf2 transcription level.

Conclusion:

These results suggested that AhR regulated Nrf2 through a direct and indirect pathway in RPE cells. This Nrf2/AhR axis mechanism may be an innovative way to provide a potential treatment for AMD by regulating AhR to increase Nrf2 dependent protective effects. 